\title{
Passing the baton of trust
}

\author{
Author: Catherine Millington-Sanders ${ }^{\mathrm{A}}$
}

Proactive, shared decision making between key professionals and sensitive communication with patients, and those important to them, can never be overestimated. Projected annual deaths in England and Wales are set to rise, highlighting the increasing need for high quality palliative and end-of-life care. The Royal College of General Practitioners and Marie Curie have worked in partnership to develop the Daffodil Standards, an evidenced-based, structured approach for practices using quality improvement methodology to help improve care for patients at an advanced stage of serious illness or approaching end of life.

KEYWORDS: Shared decision making, standards, communication, palliative and end of life, advance care planning

\section{Introduction}

With increasing pressures on clinicians in all settings, communication is a crucial tool. But what does that really mean? As a GP, on a drizzly Saturday out-of-hours shift, it can mean everything. I'm called to visit Frank, a retired teacher with deteriorating advanced chronic obstructive pulmonary disease (COPD) and severe difficulty breathing - enough to make you feel it. He recently came out of a two-week spell in hospital and his wife tells me, 'Frank said at the time, he never wants to go back!' She hands me a copy of the discharge summary, poignantly saying, 'It should all be in here, doctor.' I look down and see the words 'repeat admission with exacerbation of severe COPD'. I turn this important record over, hopeful...drugs, allergies, smoking history. I scan for any details of escalation of treatment or patient wishes. 'Do not attempt cardiopulmonary resuscitation' (DNACPR)? Advance Care Plan? A last look back in the envelope leaves me realising I'm in the dark.

The truth is, we're all in this pressure cooker of a system together. But undeniably, a hospital admission is an opportunity to open up discussions with patients and those important to them on what they would prefer to happen if they find themselves at risk of going to hospital again. For transitions of care between interfaces, passing the baton of trust by documenting and appropriately sharing these discussions can inevitably help the team win the race for the patient and those important to them.

Author: ${ }^{A}$ RCGP and Marie Curie National end-of-life care clinical champion, Royal College of General practitioners, London, UK

\section{Projected trends in palliative and end-of-life care}

With projected annual deaths in England and Wales set to increase, a recent study by Etkind et al described the morbidity trends observed from 2006 to 2014. ${ }^{1}$ The principle drivers of need for palliative care will probably be dementia, set to increase from 59,199 to 219,409 deaths per year, and cancer, which is also increasing from 143,638 to 208,636 deaths by 2040 . The new projections put the increase in number of people requiring palliative care from 2014 to 2040 at $42.4 \%$. So 161,842 more people per year, a total of 537,240 will require palliative care in 2040. 'Even this calculation does not take into account the likely increased survival and therefore duration of care that the new comorbidities will generate.

\section{Royal College of General Practitioners and Marie Curie Daffodil Standards}

Over the past three years the Royal College of General Practitioners (RCGP) and Marie Curie partnership has focused on end-of-life care as a clinical priority in order to promote and enable patient-centred, high-quality palliative and end-of-life care. There is a recognised need to support training for professionals across settings.

In this context, the RCGP and Marie Curie partnership has developed the UK General Practice core standards for advanced serious illness and end-of-life care - or 'Daffodil Standards', which will soon be piloted at selected GP practices throughout the UK. The voluntary Daffodil Standards have been developed in conjunction with a wide range of health and care experts, commissioners, patient and carer groups; they are evidence-based, peer-reviewed, and offer a structured approach for practices using quality improvement methodology to improve care for patients at an advanced stage of serious illness or approaching end of life.

The Standards outline eight core domains in which GP practices can look to attain the highest possible standards to ensure compassionate, safe and effective care, with free tools to help achieve step-by-step improvements in each area.

One feature of the Daffodil Standards is to explore how general practice can partner with general medical colleagues, particularly at interfaces of care settings. Given the variation in multidisciplinary team working in general practice, this gives an opportunity for practices and specialists to reflect together on their practice. As it is recognised that each practice is individual and resources in areas vary, the Standards do not state what 'has to be in place' and instead offer methodology for how to improve practice. The Standards, alongside the associated tools and 
resources support practices to make year-on-year improvements whatever the starting level.

\section{Top tips on care of the dying adult}

One such resource available to facilitate implementation of the Standards is Top tips on care of the dying adult in the last days of life, endorsed by the National Institute for Health and Care Excellence (NICE) and the RCGP and Marie Curie partnership, and based on NICE guidance NG31. 2,3

The 'Top tips' offer a summary of the guidance on the clinical care of adults who are dying during the last 2 to 3 days of life. These highlight top tips from the guideline recommendations, covering:

> recognising when people are entering the last few days of life

$>$ communicating and shared decision making

$>$ clinically assisted hydration

> medicines for managing pain, breathlessness, nausea and vomiting, anxiety, delirium, agitation and noisy respiratory secretions

$>$ anticipatory prescribing.

It is essential that implementation of the Standards in this field are rigorously tested and evaluated. Initially, the Standards are being piloted at selected GP practices throughout the UK in order to examine validity and feasibility, prior to wider implementation and evaluation.

\section{Conflict of interest}

The author's role as is RCGP and Marie Curie National End of Life Care Clinical Champion is funded by Marie Curie.

\section{References}

1 Etkind SN, Bone AE, Gomes B et al. How many people will need palliative care in 2040? Past trends, future projections and implications for services. BMC Medicine 2017;15:102.

2 National Institute for Health and Care Excellence. Care of the dying adult in the last days of life. NICE guidance NG31. NICE, 2015. Available online at https://www.nice.org.uk/guidance/ng31 [Accessed 20 October 2017]

3 Royal College of General Practitioners. Palliative and end-of-life care toolkit. www.rcgp.org.uk/clinical-and-research/toolkits/palliative-andend-of-life-care-toolkit.aspx

Address for correspondence: Dr Catherine Millington-Sanders, Royal College of General Practitioners, 30 Euston Road, London NW1 2FB, UK.

Email: c.millington-sanders@nhs.net

'This landmark report lays out in the starkest terms yet the devastating impact air pollution is having on our health, our economy and our society as a whole.'

\section{Every breath we take The lifelong impact of air pollution}

This major report plainly sets out the dangerous impact that air pollution has on our nation's health. Compiled by experts in medicine and environmental sciences, the report discusses the current evidence and draws up recommendations for action.

ISBN 978-1-86016-567-2 £15 including $p+p$ or free to download

Royal College of Physicians
Download the report: rcplondon.ac.uk/pollution

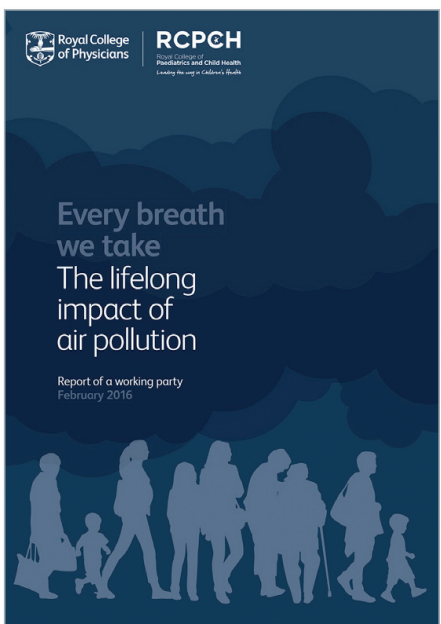

Order a copy: shop.rcplondon.ac.uk 